\title{
Effects of and satisfaction with short message service reminders for patient medication adherence: a randomized controlled study
}

\author{
Hsiu-Ling Huang ${ }^{1,2}$, Yu-Chuan Jack Li ${ }^{3}$, Yueh-Ching Chou ${ }^{4}$, Yow-Wen Hsieh ${ }^{5}$, Frank Kuo ${ }^{6}$, Wen-Chen Tsai ${ }^{\text {* }}$,
} Sinkuo Daniel Chai ${ }^{1}$, Blossom Yen-Ju Lin ${ }^{1}$, Pei-Tseng Kung ${ }^{7}$ and Chia-Jung Chuang

\begin{abstract}
Background: Medication adherence is critical for patient treatment. This study involved evaluating how implementing Short Message Service (SMS) reminders affected patient medication adherence and related factors.

Methods: We used a structured questionnaire to survey outpatients at three medical centers. Patients aged 20 years and older who were prescribed more than 7 days of a prescription medication were randomized into SMS intervention or control groups. The intervention group received daily messages reminding them of aspects regarding taking their medication; the control group received no messages. A phone follow-up was performed to assess outcomes after 8 days. Data were collected from 763 participants in the intervention group and 435 participants in the control group.

Results: After participants in the intervention group received SMS reminders to take medication or those in the control group received no messages, incidences of delayed doses were decreased by 46.4 and $78.8 \%$ for those in the control and intervention groups, respectively. The rate of missed doses was decreased by $90.1 \%$ for participants in the intervention group and $61.1 \%$ for those in the control group. We applied logistic regression analysis and determined that participants in the intervention group had a 3.2-fold higher probability of having a decrease in delayed doses compared with participants in the control group. Participants in the intervention group also showed a 2.2-fold higher probability of having a decrease in missed doses compared with participants in the control group. Conclusions: Use of SMS significantly affected the rates of taking medicine on schedule. Therefore, daily SMS could be useful for reminding patients to take their medicine on schedule.
\end{abstract}

Keywords: Short message service (SMS), Medication reminders, Personal medication platform, Patient compliance

\section{Background}

Poorly treated chronic diseases both increase health care costs and reduce patient quality of life [1]. A report published by the World Health Organization (WHO) in 2003 indicated that effective and innovative strategies for improving medication adherence can more significantly influence human health compared with advancements in medical techniques [2].

Patients often forget or delay their consumption of medication or neglect the instructions of healthcare providers.

\footnotetext{
* Correspondence: wtsai@mail.cmu.edu.tw

${ }^{1}$ Department of Health Services Administration, China Medical University, 91, Hsueh-Shih Road, Taichung 40402, Taiwan

Full list of author information is available at the end of the article
}

According to a literature review, an average of $48-80 \%$ of patients with chronic psychiatric diseases adhered to their prescribed treatment [3]. An average of $25 \%$ of diabetes patients and $53 \%$ of hypertensive patients adhered to their prescribed treatment for 6 months [4]. Such low medication adherence by various patient groups with chronic diseases has compelled the worldwide medical community to increasingly focus on applying technology to remedy this situation.

Regarding case management, using phone follow-ups improves the clinical symptoms of patients, facilitating the early identification of complications and enhancing patient adoption of healthy lifestyles [5]. The WHO recommended implementing an innovative service model 
not limited to face-to-face services to manage chronic diseases; they recommended that cell phones be used to provide timely services [6]. In developed countries, healthcare institutions often use communication technology to remind patients of follow-up appointments, generating a positive image of the healthcare institution and strengthening patient loyalty. Text messaging, also known as Short Message Service (SMS), is a simple and cost-effective tool for providing medication reminders that has been employed by several healthcare services $[7,8]$. Studies have noted positive changes when an SMS reminder was used to increase adherence to treatment programs [8-10]. Other studies showed positive effects on health-related behaviors regarding SMS interventions $[1,11]$.

We studied the effectiveness of SMS reminders as an intervention to determine whether they improved patient medication adherence. The primary aim was to determine how SMS medication reminders reduced delayed and missed medications doses; the secondary aim included determining patient satisfaction with and demand for SMSbased interventions.

\section{Methods}

\section{Study design}

The study was conducted between November 1, 2010 and October 31, 2011. After we obtained approval from the Institutional Review Boards of the study hospitals (T.V.G.H., 201006021IC; C.M.U.H., 98-08-01A; and W.H., 100008), hospital pharmacists randomly assigned two patients to the intervention group and one to the control group when patients met the inclusion criteria. We used the systematic sampling method to assign qualified patients to each group. Odd-numbered or even-numbered patients from the pharmacy department registration were assigned to each group. Subsequently, trained interviewers conducted one-on-one interviews with the potential participants, explaining the research purpose and methods in outpatient settings such as the outpatient dispensary waiting area. After agreeing to participate in the study, the patients completed a questionnaire-based pretest.

Patients in the intervention group received SMS reminders reminding them to take their medication at specific times for 7 days beginning on the second day of enrollment, and could not choose the times the reminders were sent. On the eighth day after completing the intervention we conducted a phone-based survey to assess patient medication compliance and demand for and satisfaction with the "text message medication reminder service." Figure 1 shows the study screening flowchart.

\section{Participants}

Inclusion criteria for participants were: (a) literate patients aged 20 years or older able to communicate with the investigators; (b) having a prescription longer than

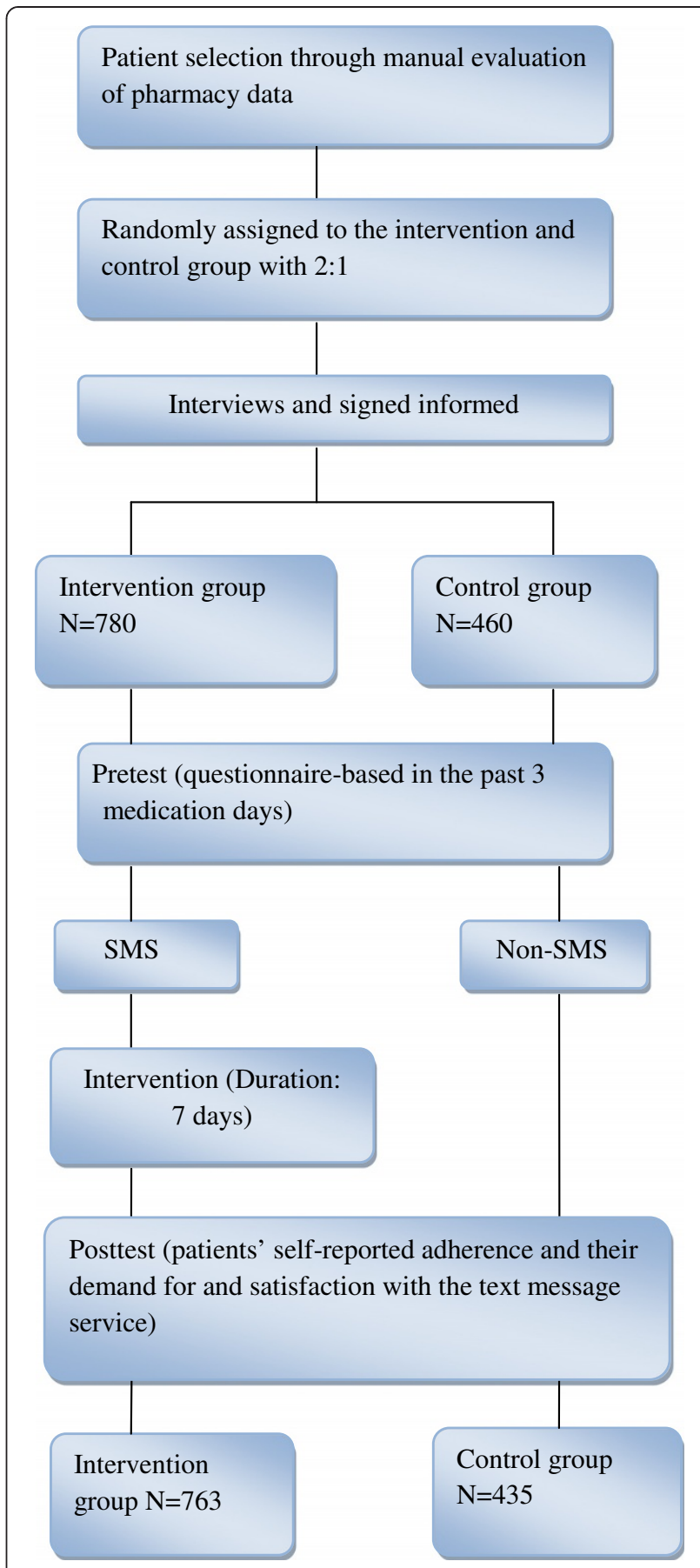

Figure 1 The SMS study screening flowchart.

7 days; (c) possessing a cell phone and knowing how to receive text messages; (d) and having at least one medication nonadherence (delayed or missed dose) experience in the past 3 days of taking medicine.

We recruited participants from three medical centers based on the large outpatient volume in each hospital. Because we focused on the effect of using SMS in the intervention group, we deliberately increased the sample 
size of this group. The sample sizes of the intervention and control groups were allocated at a ratio of 2:1.

\section{Intervention}

After patients signed and submitted informed consent forms, they completed a questionnaire-based pretest. We then collected patients' cell phone numbers to facilitate convenient post-test phone interviews. Patients' identities were recognized using hospital information system (HIS) databases; their prescriptions were obtained from the clinical department they visited during recruitment and exported to the SMS system for sending reminders. Beginning on the following day, medication reminders were sent to participants by using the SMS system; text messages were sent only to participants in the intervention group.

The SMS content depended on participants' medication and frequency of medication use. If a participant used multiple medications, he or she received reminders for each. The messages comprised varying content such as visitation date, hospital name, medication name, dosing frequency, dose, and administration methods. The first message contained complete medication information. For example, "A warm reminder! You visited the $\mathrm{xx}$ clinic at $\mathrm{xx}$ Hospital on 2010/3/15. We would like to remind you to take your prescription, which includes Panadol $^{\circ}$ (one tablet per day for 7 days), Lasix ${ }^{\bullet}$ ( 0.5 tablet per day immediately following a meal for 7 days), and Lontex ${ }^{\circ}(0.5$ tablet once per day for 7 days). Please remember to take this medication on time. We wish you a prompt recovery." The second and subsequent messages contained only simple medication reminders.

\section{Measurements}

The research tools comprised an investigator-designed questionnaire that involved a pretest and post-test. The pretest comprised basic demographic questions (e.g., sex, age, and education level), medical conditions, and selfreported medication use experiences (frequency of daily medication use, delayed doses, or missed doses during the previous 3 days). If participants did not take medicine and passed more than a half-period of time between two doses, we defined it as a "missed dose." For example, if participants needed to take medicine twice a day (i.e., once every 12 hours), and took medicine more than 6 hours late, it qualified as a missed dose. If participants did not take medication as prescribed regarding timing or dosage, but took the medicine within 6 hours, we defined it as a "delayed dose."

The post-test questionnaire was conducted by phone interview on the eighth day after the study. The post-test questions comprised structured items for the participants' self-reported adherence to medication during the previous 3 days and their demand for and satisfaction with the
SMS medication reminder service. Open-ended questions were also included to obtain participants' recommendations regarding the service. The interviewers also asked the participants whether they would recommend the service to their family or friends.

To verify the validity of the questionnaire, five experts performed a content validity test, evaluating whether the questionnaire items conformed to the study topic. The average content validity index (CVI) obtained was 0.9. Regarding reliability, the Cronbach's $\alpha$ coefficient for internal consistency reliability was 0.9 , indicating good questionnaire reliability.

\section{Data analysis}

We used SAS statistical software to manage and analyze the data obtained from the questionnaires and descriptive statistics to determine the frequency, percentage, and mean values of each demographic variable. Regarding inferential statistics, we performed chi-square tests to identify differences between the groups regarding improvements in medication adherence and the demand for and satisfaction with the SMS medication reminder service. We used McNemar's test to compare the pretest and post-test data within each group to examine distinct decreases in the incidence of delayed or missed medication doses before and after the intervention. Because the number of participants recruited from the three study hospitals differed and the management context and service model of the hospital at which most participants were recruited could affect the results of the intervention, we used weighted logistic regression to examine the factors that influenced improvements in the incidence of delayed or missed doses. We used odds ratios (OR) to describe the effect sizes.

\section{Results}

\section{Baseline data}

The intervention group and control group comprised 780 and 460 participants at the time of the pretest, respectively. The number of valid participants who completed both surveys was 763 in the intervention group and 435 in the control group. We obtained 1,198 total questionnaires from the three study hospitals. In each hospital, we collected 188, 225, and 350 questionnaires for the intervention group and 117, 118, and 200 questionnaires for the control group.

We collected data related to the patient medication and basic demographic characteristics such as sex, age, and education level (Table 1). According to the pretest results, $88.1 \%$ of the patients in the control group reported infrequently missing a medication dose, compared with $88.5 \%$ of patients in the intervention group. The proportion of patients who previously delayed a dose was 80.2 and $84.7 \%$ for the control and intervention groups, respectively. 
Table 1 Descriptive statistics of patient demographics and personal information

Variable

Control group

$\mathrm{N}=435$

Medication adherence history

Have you ever NOT adhered to medication prescriptions?

Seldom
Often

52

86

No

Yes

349

Missed doses

No

Yes

245

190

Socioeconomic status

Gender

$\begin{array}{ll}\text { Male } & 211 \\ \text { Female } & 224\end{array}$

Age (years)
$20 \sim 34$
$35 \sim 49$
$50 \sim 64$
$\geqq 65$

Education level

$\geqq$ Elementary
Junior High School
High School
College
University
Graduate school or above

Occupation

Unemployed
Military
Civil servant
Teacher
Student
Housekeeper
Self-employed
Medicine
Retired
Freelancer
Services
Worker
A.F.F.H.
Other

96
135
146
58

41

8
Intervention group

$\mathrm{N}=763$

$\%$

$\%$

$\mathrm{N}=763$

p-value

0.828

88.5

11.5

12.0

675

88

117

15.3

646

84.7

80.2

56.3

411

53.9

43.7

352

46.13

48.5

383

0.574

48.5

380

50.2

49.8

$22.1 \quad 162$

0.085

227

21.2

31.0

29.8

33.6

229

30.0

13.3

145

$<.001$

6.2

12.0

20.2

20.9

31.0

9.4

6.0

0.2

10.3

7.6

6.7

13.8

15.4

2.3

7.6

3.7

13.1

10.8

0.7

1.8
34

65

275

151

179

59

0.026

4.5

8.5

36.0

19.8

23.5

7.7
22

3

57

49

38

103

116

46

78

37

103

95

2

14
19.0

2.9

0.4

7.5

6.4

5.0

13.1

15.2

6.0

10.2

4.9

13.5

12.5

0.3

1.8

14

1.8 
Table 1 Descriptive statistics of patient demographics and personal information (Continued)

\begin{tabular}{|c|c|c|c|c|c|}
\hline \multirow[t]{2}{*}{ Variable } & \multicolumn{2}{|c|}{ Control group } & \multicolumn{2}{|c|}{ Intervention group } & \multirow[t]{2}{*}{ p-value } \\
\hline & $\mathrm{N}=435$ & $\%$ & $\mathrm{~N}=763$ & $\%$ & \\
\hline Average monthly household income & & & & & 0.025 \\
\hline$\leqq 30,000 \mathrm{NTD}$ & 94 & 21.6 & 122 & 16.0 & \\
\hline $30,001-60,000$ NTD & 110 & 25.3 & 193 & 25.3 & \\
\hline 60,001-90,000 NTD & 93 & 21.4 & 152 & 19.9 & \\
\hline $90,001-120,000$ NTD & 34 & 7.8 & 80 & 10.5 & \\
\hline $120,001-150,000$ NTD & 28 & 6.4 & 82 & 10.8 & \\
\hline$\geqq 15,0001$ NTD & 71 & 16.3 & 125 & 16.4 & \\
\hline Missing data & 5 & 1.2 & 9 & 1.2 & \\
\hline Marital status & & & & & $<.001$ \\
\hline Single & 108 & 24.8 & 178 & 23.3 & \\
\hline Married & 259 & 59.5 & 409 & 53.6 & \\
\hline Divorced/Separated & 48 & 11.0 & 156 & 20.5 & \\
\hline Widowed & 20 & 4.6 & 19 & 2.5 & \\
\hline Missing data & 0 & 0.0 & 1 & 0.1 & \\
\hline Living status & & & & & 0.735 \\
\hline Live with spouse only & 59 & 13.6 & 114 & 14.9 & \\
\hline Live with children only & 81 & 18.6 & 162 & 21.2 & \\
\hline Live with spouse and children & 138 & 31.7 & 225 & 29.5 & \\
\hline Live alone & 42 & 9.7 & 71 & 9.3 & \\
\hline Other & 115 & 26.4 & 191 & 25.0 & \\
\hline \multicolumn{6}{|l|}{ Medical history } \\
\hline Heart disease & 79 & 18.2 & 142 & 18.6 & 0.847 \\
\hline Hypertension & 120 & 27.6 & 197 & 25.8 & 0.505 \\
\hline Dialysis & 2 & 0.5 & 11 & 1.4 & 0.115 \\
\hline Stroke & 51 & 11.7 & 45 & 5.9 & 0.121 \\
\hline Peptic ulcer & 39 & 9.0 & 73 & 9.6 & 0.731 \\
\hline Thyroid disease & 42 & 9.7 & 36 & 4.7 & $<.001$ \\
\hline Chronic kidney disease & 9 & 2.1 & 13 & 1.7 & 0.651 \\
\hline Hyperlipidemia & 16 & 3.7 & 32 & 4.2 & 0.662 \\
\hline Diabetes & 88 & 20.2 & 141 & 18.5 & 0.459 \\
\hline Gynecology disease & 3 & 0.7 & 8 & 1.1 & 0.531 \\
\hline Asthma/Emphysema & 9 & 2.1 & 15 & 2.0 & 0.903 \\
\hline Urinary disease & 7 & 1.6 & 8 & 1.1 & 0.401 \\
\hline Cancer & 4 & 0.9 & 2 & 0.3 & $<.001$ \\
\hline Chronic liver disease & 20 & 4.6 & 42 & 5.5 & 0.496 \\
\hline Gout & 19 & 4.4 & 17 & 2.2 & 0.037 \\
\hline Pneumonia & 8 & 1.8 & 3 & 0.4 & 0.012 \\
\hline Mental or psychiatric disease & 14 & 3.2 & 11 & 1.4 & 0.039 \\
\hline Immunity disease & 3 & 0.7 & 12 & 1.6 & 0.186 \\
\hline Others & 105 & 24.1 & 190 & 24.9 & 0.768 \\
\hline
\end{tabular}


The proportion of patients who previously missed a dose was 43.7 and $46.1 \%$, for the control and intervention groups, respectively. The pretest showed no significant differences regarding medication use experience between the groups $(\mathrm{P}>0.05)$. Regarding basic demographic characteristics, sex, age, and living status did not significantly differ between the groups $(P>0.05)$. Regarding chronic diseases, heart disease, diabetes, and hypertension were the most common conditions for patients in both groups. Most of the chronic diseases listed in Table 1 demonstrated similar distribution in both groups.

\section{The link between the SMS intervention and medication adherence}

Comparing results before and after the SMS intervention (Table 2) shows that $78.8 \%$ of the participants in the intervention group had a decreased (i.e., no delayed doses) incidence of delayed doses; this figure was $46.4 \%$ for the control group. The differences between the pretest and post-test were significant for both groups $(\mathrm{P}<0.05)$. Regarding missed doses, $90.1 \%$ of the participants in the intervention group showed a decrease (i.e., no missed doses), whereas only $61.1 \%$ of the participants in the control group showed a decrease between the pretest and post-test
( $\mathrm{P}<0.05$ for both groups). Evaluating improvement regarding decreases in the frequency of delayed doses showed that 91.5 and $67.3 \%$ of participants in the intervention and control groups, respectively, who previously experienced delayed doses, demonstrated medication adherence improvement. Regarding decreases in the frequency of missed doses, 96.0 and $84.7 \%$ of participants who previously experienced missed doses, in the intervention and control groups, respectively, demonstrated medication adherence improvement (results not shown in the table). These findings suggest that SMS medication reminders effectively decreased the incidence of delayed and missed doses and enhanced patient adherence to medication.

\section{Factors that influence decreases in the incidence of delayed doses}

We used weighted logistic regression to identify the factors that affected the decrease in the incidence of delayed doses. Table 3 shows that participants in the intervention group had a 3.2 times $(95 \% \mathrm{CI}=2.7-3.7)$ higher chance of a decrease in the incidence of delayed doses compared with participants in the control group.

Regarding medical history, participants with heart disease, hypertension, stroke, and thyroid disorder were less

Table 2 Comparing the results before and after the SMS intervention

\begin{tabular}{|c|c|c|c|c|c|c|c|}
\hline & \multirow[t]{2}{*}{ Variable } & \multicolumn{2}{|c|}{ Before SMS intervention } & \multicolumn{2}{|c|}{ After SMS intervention } & \multirow{2}{*}{$\begin{array}{c}\text { Improvement } \\
\%\end{array}$} & \multirow[t]{2}{*}{ P-value } \\
\hline & & $\mathrm{N}$ & $\%$ & $\mathbf{N}$ & $\%$ & & \\
\hline & & & & & & & $<.001^{\mathrm{a}}$ \\
\hline \multirow[t]{5}{*}{ Intervention group } & Delayed doses (Total) & 646 & $84.7^{c}$ & 137 & $18.0^{c}$ & 78.8 & $<.001^{\mathrm{b}}$ \\
\hline & $20 \sim 34 \mathrm{y} / \mathrm{o}$ & 146 & 90.1 & 32 & 19.8 & 78.1 & \\
\hline & $35 \sim 49$ y/o & 181 & 79.7 & 52 & 22.9 & 71.3 & \\
\hline & $50 \sim 64 \mathrm{y} / \mathrm{o}$ & 196 & 85.6 & 35 & 15.2 & 82.2 & \\
\hline & $\geqq 65 \mathrm{y} / \mathrm{o}$ & 123 & 84.8 & 18 & 12.4 & 85.4 & \\
\hline \multirow[t]{5}{*}{ Control group } & Delayed doses (Total) & 349 & $80.2^{c}$ & 187 & $43.0^{c}$ & 46.4 & $<.001^{\mathrm{b}}$ \\
\hline & $20 \sim 34 \mathrm{y} / \mathrm{o}$ & 83 & 86.5 & 58 & 60.4 & 30.1 & \\
\hline & $35 \sim 49 \mathrm{y} / \mathrm{o}$ & 105 & 77.8 & 62 & 45.9 & 41.0 & \\
\hline & $50 \sim 64 \mathrm{y} / \mathrm{o}$ & 113 & 77.4 & 54 & 37.0 & 52.2 & \\
\hline & $\geqq 65 \mathrm{y} / \mathrm{o}$ & 48 & 82.8 & 13 & 22.4 & 72.9 & \\
\hline \multirow[t]{5}{*}{ Intervention group } & Missed doses (Total) & 352 & $46.1^{\mathrm{c}}$ & 35 & $4.6^{c}$ & 90.1 & $<.001^{\mathrm{b}}$ \\
\hline & $20 \sim 34 \mathrm{y} / \mathrm{o}$ & 90 & 55.6 & 9 & 5.6 & 90.0 & \\
\hline & $35 \sim 49 \mathrm{y} / \mathrm{o}$ & 97 & 42.7 & 9 & 4.0 & 90.7 & \\
\hline & $50 \sim 64 \mathrm{y} / \mathrm{o}$ & 101 & 44.1 & 10 & 4.4 & 90.0 & \\
\hline & $\geqq 65 \mathrm{y} / \mathrm{o}$ & 64 & 44.1 & 7 & 4.8 & 89.1 & \\
\hline \multirow[t]{5}{*}{ Control group } & Missed doses (Total) & 190 & $43.7^{c}$ & 74 & $17.0^{c}$ & 61.1 & $<.001^{\mathrm{b}}$ \\
\hline & $20 \sim 34 \mathrm{y} / \mathrm{o}$ & 52 & 54.2 & 28 & 29.2 & 46.2 & \\
\hline & $35 \sim 49 \mathrm{y} / \mathrm{o}$ & 62 & 45.9 & 14 & 10.4 & 77.4 & \\
\hline & $50 \sim 64$ y/o & 52 & 35.6 & 30 & 20.6 & 42.3 & \\
\hline & $\geqq 65 \mathrm{y} / \mathrm{o}$ & 24 & 41.4 & 2 & 3.5 & 91.7 & \\
\hline
\end{tabular}

a: used chi-square test to examine the difference between groups; b: Used the McNemar's test to examine the difference between the pre-test and post-test data within the group. c: intervention group $\%=n / 763$; control group $\%=n / 435$. 
Table 3 Weighted logistic regression of SMS on decreasing delayed doses

\begin{tabular}{l} 
Variable \\
\hline After SMS interventio \\
No (reference) \\
Yes \\
Socioeconomic status \\
Gender \\
Male (reference) \\
Female \\
Age (years) \\
$20 \sim 34$ (reference) \\
$35 \sim 49$ \\
$50 \sim 64$ \\
$\geqq 65$
\end{tabular}

Education level

Elementary or less (reference)

Junior High School

High School

College

University

Graduate school or above

Occupation

Unemployed (reference)

Military

Civil servant

Teacher

Student

Housekeeper

Self-employed

Medicine

Retired

Freelancer

Services

Worker

A.F.F.H.

Other

$\leqq 30,000$ NTD (reference)

30,001-60,000 NTD

60,001-90,000 NTD

90,001-120,000 NTD

120,001-150,000 NTD

$\geqq 150,001$ NTD

Marital status

Single (reference)

Married

Divorced/Separated

\section{OR}

95\% Cl P-value

$3.2^{* *} \quad 2.7 \quad 3.7<.001$

$\begin{array}{lllll}0.6 & * * & 0.4 & 0.8 & 0.001 \\ 0.9 & & 0.6 & 1.2 & 0.419 \\ 1.0 & & 0.6 & 1.5 & 0.955\end{array}$

0.955

$\begin{array}{lllll}1.1 & & 0.7 & 1.7 & 0.641 \\ 2.1 & * * & 1.4 & 3.2 & <.001 \\ 1.3 & & 0.8 & 1.9 & 0.288 \\ 0.9 & & 0.6 & 1.5 & 0.782 \\ 0.6 & & 0.4 & 1.1 & 0.076\end{array}$

$\begin{array}{llll}0.6 & 0.4 & 1.1 & 0.076\end{array}$

0.4 .1 .1076

$\begin{array}{lllll}1.4 & & 0.4 & 5.3 & 0.582 \\ 0.9 & & 0.5 & 1.4 & 0.579 \\ 2.1 & * & 1.2 & 3.6 & 0.011 \\ 1.0 & & 0.6 & 1.7 & 0.938 \\ 0.6 & * & 0.4 & 0.9 & 0.023 \\ 0.9 & & 0.6 & 1.4 & 0.611 \\ 1.8 & & 1.0 & 3.5 & 0.064 \\ 0.7 & & 0.4 & 1.1 & 0.145 \\ 0.7 & & 0.4 & 1.2 & 0.234 \\ 1.2 & & 0.8 & 1.8 & 0.418 \\ 0.7 & & 0.4 & 1.1 & 0.078 \\ 2.7 & & 0.8 & 9.7 & 0.127 \\ 1.1 & & 0.5 & 2.1 & 0.881 \\ & & & & \\ 0.8 & * & 0.6 & 1.0 & 0.043 \\ 1.6 & * * & 1.2 & 2.1 & 0.001 \\ 0.8 & & 0.6 & 1.2 & 0.254 \\ 0.7 & & 0.5 & 1.0 & 0.062 \\ 2.1 & * * & 1.5 & 3.0 & <.001\end{array}$

$\begin{array}{lllll}1.7 & * * & 1.2 & 2.4 & 0.005\end{array}$

$\begin{array}{llll}0.7 & 0.5 & 1.1 & 0.143\end{array}$
Table 3 Weighted logistic regression of SMS on decreasing delayed doses (Continued)

Widowed

0.7

0.231

Living status

Live with spouse only(reference)

$\begin{array}{lllll}2.7 & * * & 1.8 & 4.1 & <.001 \\ 0.7 & * * & 0.5 & 0.9 & 0.006 \\ 0.7 & * & 0.4 & 1.0 & 0.049 \\ 1.3 & & 0.9 & 1.9 & 0.149\end{array}$

Medical history

\begin{tabular}{llllll} 
Heart disease & 0.7 & $* *$ & 0.5 & 0.9 & 0.001 \\
Hypertension & 0.7 & $* *$ & 0.6 & 0.9 & 0.001 \\
Dialysis & 0.6 & & 0.3 & 1.3 & 0.217 \\
Stroke & 0.5 & $* *$ & 0.4 & 0.7 & $<.001$ \\
Peptic ulcer & 1.8 & $* *$ & 1.2 & 2.5 & 0.002 \\
Thyroid disease & 0.6 & $* *$ & 0.4 & 0.8 & 0.001 \\
Chronic kidney disease & 0.7 & & 0.4 & 1.3 & 0.285 \\
Hyperlipidemia & 1.0 & & 0.7 & 1.5 & 0.876 \\
Diabetes & 1.0 & & 0.8 & 1.3 & 0.930 \\
Gynecology disease & 0.9 & & 0.4 & 1.8 & 0.672 \\
Asthma/Emphysema & 0.8 & & 0.4 & 1.3 & 0.328 \\
Urinary disease & 1.0 & & 0.5 & 2.0 & 0.992 \\
Cancer & 3.9 & $*$ & 1.0 & 14.8 & 0.046 \\
Chronic liver disease & 1.3 & & 0.9 & 2.1 & 0.199 \\
Gout & 1.3 & & 0.8 & 2.1 & 0.357 \\
Pneumonia & 2.5 & & 0.9 & 7.0 & 0.090 \\
Mental or psychiatric disease & 1.0 & & 0.6 & 1.6 & 0.884 \\
Immunity disease & 3.9 & $*$ & 1.67 & 9.0 & 0.002 \\
Others & 0.52 & 0.8 & $<.001$ \\
\hline
\end{tabular}

$\mathrm{N}=1198$. Dependent variable: 1 indicates with complete improvement; 0 indicates without complete improvement. Medical history reference group is the participants without this disease; A.F.F.H: agricultural, forestry, fishery or husbandry. ${ }^{*} \mathrm{p}<0.05 ;{ }^{* *} \mathrm{p}<0.01$

likely to have a decreased incidence of delayed doses compared with participants who lacked such diseases. However, participants with peptic ulcers, cancer, or immunological diseases were significantly more likely to experience a decrease in the incidence of delayed doses compared with participants who lacked such diseases.

Factors that influence reduced incidence of missed doses The weighted logistic regression results for factors that influenced the decrease in the incidence of missed doses showed that participants in the intervention group had a 2.2-fold (95\% CI = 1.9-2.6) higher likelihood of experiencing a decrease compared with participants in the control group (Table 4). Additionally, women were significantly more likely to demonstrate improvement compared with 
Table 4 Weighted logistic regression of SMS on decreasing missed doses

\begin{tabular}{llll}
\hline Variable & OR & $95 \% \mathrm{Cl}$ & P-value \\
\hline
\end{tabular}

After SMS intervention

No (reference)

Yes

$2.2 \quad * * \quad 1.9 \quad 2.6<.001$

\section{Socioeconomic status}

Gender

Male (reference)

Female

Age (years)

$20 \sim 34$ (reference)
$35 \sim 49$
$50 \sim 64$
$\geqq 65$

Education level

Elementary or less (reference)

Junior High School

High School

College

University

Graduate school or above

Occupation

Unemployed (reference)

Military

Civil servant

Teacher

Student

Housekeeper

Self-employed

Medicine

Retired

Freelancer

Services

Worker

A.F.F.H.

Other

Average monthly household income

$\leqq 30,000$ NTD(reference)

30,001-60,000 NTD

60,001-90,000 NTD

90,001-120,000 NTD

120,001-150,000 NTD

$\geqq 150,001$ NTD

Marital status

Single (reference)

Married
$2.1 \quad * * \quad 1.8 \quad 2.6<.001$

$\begin{array}{lllll}0.7 & * & 0.5 & 1.0 & 0.024 \\ 0.7 & * & 0.5 & 0.9 & 0.014 \\ 0.6 & * & 0.4 & 0.9 & 0.009\end{array}$

Table 4 Weighted logistic regression of SMS on decreasing missed doses (Continued)

Divorced/Separated

$\begin{array}{lllll}0.4 & * * & 0.3 & 0.6 & <.001 \\ 1.7 & & 1.0 & 2.9 & 0.054\end{array}$

Widowed

Living status

Live with spouse only (reference)

Medical history

$\begin{array}{llll}0.7 & 0.5 & 1.1 & 0.093\end{array}$

Live with spouse and children

Live alone

Other

$1.4 * \quad 1.1 \quad 1.8 \quad 0.013$

Medical history

Heart disease

Hypertension

Dialysis

Stroke

Peptic ulcer

Thyroid disease

Chronic kidney disease

Hyperlipidemia

Diabetes

Gynecology disease

Asthma/Emphysema

Urinary disease

Cancer

Chronic liver disease

Gout

Pneumonia

Mental or psychiatric disease

Others

$\mathrm{N}=1$ 198. Dependent variable: 1 indicates with complete improvement: 0 indicates without complete improvement. Medical history reference group is the participants without this disease. The patients with immunity disease had no decrease on missed doses; A.F.F.H: agricultural, forestry, fishery or husbandry. ${ }^{*} \mathrm{p}<0.05 ;{ }^{*} \mathrm{p}<0.01$.

men $(\mathrm{OR}=2.1 ; 95 \% \mathrm{CI}=1.8-2.6)$. Participants with heart disease, hypertension, stroke, peptic ulcers, and thyroid disorder were less likely to show a decrease in the incidence of missed doses compared with participants who lacked such diseases. However, participants with psychiatric disorders $(\mathrm{OR}=2.2,95 \% \mathrm{CI}=1.3-3.8)$ were more likely to have a decrease in the incidence of delayed doses compared with participants who lacked such disorders.

\section{Impression of and satisfaction with the SMS intervention} Table 5 shows participants' satisfaction with the SMS intervention; the mean satisfaction score was 4.3 out of 5 . All satisfaction items scored at least 3 points, and the items "precision of wording," "understandability of the 
Table 5 Satisfaction with and demand for the SMS content

\begin{tabular}{lcc}
\hline Satisfaction with SMS content $(\mathbf{N}=\mathbf{7 6 3})$ & Weighted mean & SD \\
\hline The SMS clearly describes the frequency of medication use. & 3.8 & 0.7 \\
The SMS clearly describes the method of medication use. & 3.1 & 0.7 \\
Frequency of SMSs received & 4.2 & 1.0 \\
Satisfaction with the precision of wording in the SMS & 4.2 & 0.9 \\
Satisfaction with the understandability of the content of the SMS & 4.2 & 0.8 \\
Satisfaction with medication use privacy in the SMS & 4.3 & Weighted mean \\
Overall satisfaction with the SMS & 2.8 & 0.9 \\
\hline Demand for SMS content (N= 763) & 2.5 & SD \\
\hline Demand for SMS clearly displaying the frequency of medication & 2.9 & 0.9 \\
Demand for SMS clearly showing method of medication & 2.7 \\
Demand for SMS clearly displaying the medication dose & 0.9 \\
Frequency of SMSs received & 0.9 \\
\hline
\end{tabular}

SMS: Short message service.

A 5-point Likert scale, ranging from 1, not at all necessary/extremely unsatisfied, to 5, highly necessary/extremely satisfied.

content," and "medication use privacy" scored more than 4 points. Regarding participant demand for the SMS intervention, "clearly displaying the medication dose" received the highest score, followed by "clearly displaying the frequency of dose."

Of the participants who received SMS reminders (Table 6), 93.7\% of the participants considered it unnecessary for a reminder to be resent after the time at which the medication should have been consumed. Additionally, $91.6 \%$ of participants in the intervention group reported a willingness to recommend the SMS service to their family and friends. In the intervention group, $83.1 \%$ of participants believed that the SMS intervention was helpful for preventing missed or delayed doses, and
73.7\% of participants considered the service beneficial for disease management. However, $7.1 \%(\mathrm{~N}=54)$ of participants indicated that the SMS did not help reduce delayed or missed doses; thus, we performed a subgroup analysis and determined that most of these participants were women $(\mathrm{N}=39,72.2 \%)$, married, and housekeepers.

\section{Discussion}

The study intervention involved using SMS to remind patients to consume their medication. We evaluated patients' medication adherence behavior and satisfaction with and demand for an SMS reminder service after the intervention. The SMS intervention significantly decreased the incidence of delayed and missed doses among participants

Table 6 Demand for SMS reminders

\begin{tabular}{|c|c|c|c|c|c|}
\hline Variable & $N=763$ & $\%$ & Variable & $N=763$ & $\%$ \\
\hline \multicolumn{3}{|c|}{$\begin{array}{l}\text { Preferring time to receive text messages before } \\
\text { medication taken }\end{array}$} & \multicolumn{3}{|c|}{$\begin{array}{l}\text { Helpfulness of the text message medication reminder in improving } \\
\text { the incidence of delayed or missed doses }\end{array}$} \\
\hline 10 minutes & 262 & 34.3 & Very unhelpful & 13 & 1.7 \\
\hline 15 minutes & 65 & 8.5 & Unhelpful & 41 & 5.4 \\
\hline 30 minutes & 337 & 44.2 & Neutral & 75 & 9.8 \\
\hline 60 minutes & 71 & 9.3 & Helpful & 401 & 52.6 \\
\hline others & 28 & 3.7 & Very helpful & 233 & 30.5 \\
\hline \multicolumn{3}{|c|}{$\begin{array}{l}\text { Necessity of receiving text messages after the time medication } \\
\text { should be consumed }\end{array}$} & \multicolumn{3}{|c|}{ Benefits of text message medication reminders for disease control } \\
\hline Yes & 48 & 6.3 & Very unhelpful & 14 & 1.8 \\
\hline No & 715 & 93.7 & Unhelpful & 55 & 7.2 \\
\hline \multicolumn{3}{|c|}{$\begin{array}{l}\text { Willingness to recommend the text message service to family } \\
\text { and friends }\end{array}$} & Neutral & 132 & 17.3 \\
\hline Yes & 699 & 91.6 & Helpful & 373 & 48.9 \\
\hline No & 64 & 8.4 & Very helpful & 189 & 24.8 \\
\hline
\end{tabular}


in the intervention group, who showed a $29.0 \%$ decrease in the incidence regarding missed doses and $32.4 \%$ for the decrease in the incidence of delayed doses. These findings were similar to those reported by da Costa [8], who evaluated the effectiveness of and patient satisfaction with an SMS service that reminded women in Brazil who were diagnosed with AIDS to consume anti-viral drugs. Other studies have reported similar results, indicating that SMS reminders help patients consume their medications on time and reduce the incidence of delayed doses [12,13].

In the current study, the rates for the decreases in the incidence of delayed doses for the participants in the control and intervention groups were 46.4 and $78.8 \%$, respectively; these figures were 61.1 and $90.1 \%$, respectively, for decreases in the incidence of missed doses. This indicates that participants in the control group also experienced significant improvement in medication adherence. This could result from the Hawthorne effect or increased efficacy in self-managing their illnesses [14]. Previous studies have shown that in the healthcare field text message reminders were effective in increasing patient attention to treatment, decreasing the incidence of missed doses, and enhancing medication adherence [8,15].

We followed up with participants after only 7 days; however, long-term adherence is more difficult to maintain than is short-term adherence. Hanauer et al. used e-mail and SMS reminders to support diabetes management and identified decreases in SMS use after 2 months and 3 months [16]. Another study of SMS in adults with diabetes showed no change in blood glucose measurement activity throughout the 1-year study period [17]. Therefore, SMS messaging may be more appropriate for use with medications, such as some antibiotics, taken in the short term, compared with long-term medications used to treat chronic diseases.

\section{SMS improves medication adherence}

We used weighted logistic regression to examine the factors that influence improvement in the incidence of delayed and missed medication doses. The results showed that the SMS intervention significantly decreased the incidence of delayed and missed doses. This was consistent with a previous finding that indicated SMS interventions enabled patients to consume their medication on time [18]. Regarding age (Table 4), participants aged 65 years or older were significantly less likely to experience decreases in the incidence of missed doses compared with those aged $20-34$ years $(\mathrm{OR}=0.6,95 \% \mathrm{CI}=0.4-0.9)$. According to related literature, senior patients are often more resistant to behavioral change and are likely to stop taking medication based on personal decisions [19]. In addition, senior patient may be less familiar with cell phones compared with younger patients, increasing their likelihood of ignoring text message reminders, thus limiting decreases in the incidence of missed doses. However, comparing Tables 3 and 4 shows that when senior participants neglected or delayed taking medication, after they received SMS reminders they had a higher likelihood of taking medication than did those who missed taking the medication. This suggests that if senior participants delayed taking medication, when they received reminders they had a higher likelihood of improving their adherence than did those who lacked a strong intention to take their medication and missed taking it despite the reminder. Military personnel, civil servants, teachers, and students demonstrated similar behaviors. These results were similar with those of a previous study [20].

Regarding occupation, military personnel, civil servants, teachers, and students were less likely to experience decreases in the incidence of missed doses compared with unemployed participants. This could be because the military personnel, civil servants, teachers, and students in our study were typically young or middle-aged adults who demonstrated relatively better health compared with unemployed participants; therefore, these participants might consider long-term medication use unnecessary.

Regarding medical history, participants with hypertension, heart disease, stroke, or thyroid disease were less likely to experience decreases in the incidence of delayed or missed doses compared with participants who lacked such diseases. This finding was consistent with that reported by a previous study [20]. This study indicated that hypertensive patients tended to have confidence in their ability at self-control and were likely to adjust their medication consumption behavior arbitrarily, leading to poor adherence [20]. Therefore, SMS interventions may not reduce the incidence of delayed or missed doses among specific patient groups. A previous study also emphasized that patients with chronic diseases may have less motivation to consume medication regularly if their condition did not seem to improve, was incurable, or yielded side effects because of long-term medication use [21]. Thus, SMS interventions may fail to significantly alter the medication consumption behavior of patients with chronic diseases.

\section{Satisfaction with and demand for the SMS intervention}

Numerous observational studies have focused on satisfaction with and demand for SMS interventions [22-25]. In the current study, according to the results of participants' satisfaction with the SMS intervention, the item "frequency of the SMS" received the lowest satisfaction score of 3.1 points. Participants reported that "the frequency of text messages sent was too high," "text messages did not need to be sent often," and "the number of messages was excessive." These responses indicate that although the SMS intervention could help remind participants to consume medicines, it could also induce negative perceptions. Regarding participant demand for the SMS intervention, most participants (44.2\%) wished 
to receive a reminder 30 minutes before the time medication should be consumed (Table 5). However, 93.2\% of participants disliked when a message was resent after the time medication should be consumed to ensure that they would immediately consume a missed dose. Approximately $91.6 \%$ of the participants were willing to recommend the SMS intervention to their family and friends, suggesting that the SMS reminder intervention was effective. Of the participants who stated that the SMS reminders did not reduce delays or missed doses, most were women $(72.2 \%)$, married, and housekeepers. We speculate that because these participants had more flexible time to care for themselves and may already have been adhering to their medication satisfactorily, they did not perceive that the SMS intervention enhanced their medication adherence.

Previous studies that have adopted SMS interventions for increasing medication adherence all showed that patients in the intervention group experienced higher treatment effectiveness compared with control groups. This could be because SMSs pose a minimal interruption to patients' lives and are low in cost [8]. Compared with other approaches, SMS is simpler and more satisfying for users. Additionally, because the SMS reminder service can prompt patients to be responsible for their own health, it serves a vital function in healthcare services.

\section{Limitations}

Medication adherence problems are typically related to use of long-term medications for chronic diseases. In the current study, participants were monitored for only 7 days. Such a short-term follow-up might not properly interrogate the relationship between long-term medication adherence and use of SMS. Because we did not design the study to specify the medications used by participants, we could not evaluate the effects and outcome of their medication treatment. Additionally, the daily SMS system sent more than one reminder to participants who took several kinds of medications based on various medication schedules, which resulted in inconveniencing these participants. Many studies have shown that medication adherence outcome data that are purely reliant on self-reporting have a high likelihood of reporting bias [26,27]. Because overstimulation might exist in both the intervention and control groups, the current study included two groups (intervention vs. control) that completed pretests and posttests to reduce the effects of reporting bias.

\section{Conclusions}

This controlled study showed that SMS intervention enhanced patients' medication adherence. After the 7-day SMS intervention, patients in the experimental group showed greater decreases in the incidences of both delayed and missed doses compared with the control group patients. The findings of this study and those of previous studies show that use of SMS can effectively improve patient medication adherence, prompting most patients to respond favorably to such services. Therefore, an SMS reminder system is a simple, effective, and inexpensive strategy $[28,29]$. Future implementation of SMS in Chineselanguage settings could contain pictures and both Chinese and English drugs names, enabling patients to verify their use, thereby decreasing the incidence of delayed and missed doses.

\section{Abbreviations}

SMS: Short message service; PMP: Personal medication management platform; WHO: World Health Organization; HIS: Hospital information system; NTD: New TAIWAN Dollar; AIDS: Acquired immunodeficiency syndrome.

\section{Competing interests}

The authors declare that they have no competing interests.

\section{Authors' contributions}

HLH and WCT drafted the manuscript. WCT and YCL designed the study. YCC, YWH, FK, PTK and CJC collected data. SDC, PTK and BYJL were responsible for study conceptualization and developing the analytical plan. All authors read and approved the final manuscript.

\section{Acknowledgments}

This research was supported by the grants (CMU99-ASIA-18, NSC 97-3114-E010) from China Medical University, Asia University, and the National Science Council of Taiwan, ROC. The authors thank the SMS team at China Medical University Hospital, Taipei Veterans General Hospital, and Wanfang Hospital for their technical support. We would especially like to thank Dr. Yu-Chuan (Jack) Li as the main contributor to this project.

\section{Author details}

'Department of Health Services Administration, China Medical University, 91, Hsueh-Shih Road, Taichung 40402, Taiwan. ${ }^{2}$ Hospital Administration Commission, Department of Health, Executive Yuan, Taipei, Taiwan. ${ }^{3}$ Department of Graduate Institute of Biomedical Informatics, Taipei Medical University, Taipei, Taiwan. ${ }^{4}$ Department of Pharmaceutical Service, Taipei Veterans General Hospital, Taipei, Taiwan. ${ }^{5}$ Department of Pharmaceutical Service, China Medical University Hospital, Taichung, Taiwan. ${ }^{6}$ Department of Information Management, National Taiwan University, Taipei, Taiwan.

'Department of Healthcare Administration, Asia University, Taichung, Taiwan.

Received: 1 March 2013 Accepted: 13 November 2013

Published: 16 November 2013

\section{References}

1. Simpson SH, Eurich DT, Majumdar SR, Padwal RS, Tsuyuki RT, Varney J, Johnson JA: A meta-analysis of the association between adherence to drug therapy and mortality. BMJ 2006, 333(7557):15.

2. WHO: Adherence to long-term therapies. Geneva: Organisation wH; 2003.

3. Julius RJ, Novitsky MA Jr, Dubin WR: Medication adherence: a review of the literature and implications for clinical practice. J Psychiatr Pract 2009, 15(1):34-44

4. McDonald HP, Garg AX, Haynes RB: Interventions to enhance patient adherence to medication prescriptions: scientific review. JAMA 2002, 288(22):2868-2879.

5. Norris SL, Nichols PJ, Caspersen CJ, Glasgow RE, Engelgau MM, Jack L Jr, Isham G, Snyder SR, Carande-Kulis VG, Garfield S: The effectiveness of disease and case management for people with diabetes: a systematic review. Am J Prev Med 2002, 22(4):15-38.

6. Gentles SJ, Lokker C, McKibbon KA: Health information technology to facilitate communication involving health care providers, caregivers, and pediatric patients: a scoping review. J Med Internet Res 2010, 12(2):e22.

7. Cocosila M, Archer N, Brian Haynes R, Yuan Y: Can wireless text messaging improve adherence to preventive activities? Results of a randomised controlled trial. Int I Med Inform 2009, 78(4):230-238.

8. da Costa TM, Barbosa BJP, Sigulem D, de Fátima MH, Pisa IT: Results of a randomized controlled trial to assess the effects of a mobile SMS-based 
intervention on treatment adherence in HIV/AIDS-infected Brazilian women and impressions and satisfaction with respect to incoming messages. Int J Med Inform 2012, 81(4):257-269.

9. Vervloet $M$, Linn AJ, van Weert JC, de Bakker DH, Bouvy ML, van Dijk L: The effectiveness of interventions using electronic reminders to improve adherence to chronic medication: a systematic review of the literature. J Am Med Inform Assoc 2012, 19(5):696-704.

10. Lester RT, Ritvo P, Mills EJ, Kariri A, Karanja S, Chung MH, Jack W, Habyarimana J, Sadatsafavi M, Najafzadeh M: Effects of a mobile phone short message service on antiretroviral treatment adherence in Kenya (WelTel Kenya1): a randomised trial. Lancet 2010, 376(9755):1838-1845.

11. Suffoletto B, Calabria J, Ross A, Callaway C, Yealy DM: A mobile phone text message program to measure oral antibiotic use and provide feedback on adherence to patients discharged from the emergency department. Acad Emerg Med 2012, 19(8):949-958.

12. Zygmunt A, Olfson M, Boyer CA, Mechanic D: Interventions to improve medication adherence in schizophrenia. Am J Psychiatry 2002, 159(10):1653-1664.

13. Strandbygaard U, Thomsen SF, Backer V: A daily SMS reminder increases adherence to asthma treatment: a three-month follow-up study. Respir Med 2010, 104(2):166-171.

14. Jones G, Hawkins K, Mullin R, Nepusz T, Naughton DP, Sheeran P, Petroczi A: Understanding how adherence goals promote adherence behaviours: a repeated measure observational study with HIV seropositive patients. BMC Public Health 2012, 12:587.

15. Vervloet M, van Dijk L, Santen-Reestman J, Van Vlijmen B, Van Wingerden $P$, Bouvy $M L$, de Bakker DH: SMS reminders improve adherence to oral medication in type 2 diabetes patients who are real time electronically monitored. Int J Med Inform 2012, 81(9):594-604.

16. Hanauer DA, Wentzell K, Laffel N, Laffel LM: Computerized Automated Reminder Diabetes System (CARDS): e-mail and SMS cell phone text messaging reminders to support diabetes management. Diabetes Technol Therap 2009, 11(2):99-106.

17. Vähätalo M, Virtamo H, Viikari J, Rönnemaa T: Cellular phone transferred self blood glucose monitoring: prerequisites for positive outcome. Pract Diabetes Int 2004, 21(5):192-194.

18. Ollivier L, Romand O, Marimoutou C, Michel R, Pognant C, Todesco A, Migliani R, Baudon D, Boutin J-P: Use of short message service (SMS) to improve malaria chemoprophylaxis compliance after returning from a malaria endemic area. Malar J 2009, 8(236):1475-2875

19. Yu-Pei Lin Y-HH, Yi-Ching Y, Jin-Shang W, Chih-Jen C, Feng-Hwa L: Adherence to antihypertensive medications among the elderly: a community-based survey in Tainan City, Southern Taiwan. Taiwan Geriatr Gerontol 2007, 2(3):176-189.

20. Banning M: A review of interventions used to improve adherence with medication in older people. Int J Nurs Stud 2009, 46(11):1505-1515.

21. Haynes RB AE, Ackloo E, Sahota N, McDonald HP, Yao X: Interventions for enhancing medication adherence. Cochrane Database Syst Rev 2008, 2:2.

22. Krampera M, Venturini F, Benedetti F, Oliani A, Carolei S, Visco C, Aqel H, Pizzolo G, Scroccaro G: Computer-based drug management in a bone marrow transplant unit: a suitable tool for multiple prescriptions even in critical conditions. Br J Haematol 2004, 125(1):50-57.

23. Lee F, Teich JM, Spurr CD, Bates DW: Implementation of physician order entry: user satisfaction and self-reported usage patterns. J Am Med Inform Assoc 1996, 3(1):42-55.

24. Murff HJ, Kannry J: Physician satisfaction with two order entry systems. J Am Med Inform Assoc 2001, 8(5):499-509.

25. Wilson JP, Bulatao PT, Rascati KL: Satisfaction with a computerized practitioner order-entry system at two military health care facilities. Am J Health Syst Pharm 2000, 57(23):2188-2195.

26. Leroux J, Rizzo JA, Sickles R: The role of self-reporting bias in health, mental health and labor force participation: a descriptive analysis. Empir Econ 2012, 43(2):525-536.

27. Harrison L: The validity of self-reported drug use in survey research: an overview and critique of research methods. NIDA Res Monogr 1997, 167:17-36.
28. Da Costa TM, Salomão PL, Martha AS, Pisa IT, Sigulem D: The impact of short message service text messages sent as appointment reminders to patients' cell phones at outpatient clinics in Sao Paulo, Brazil. Int J Med Inform 2010, 79(1):65-70.

29. Geraghty M, Glynn F, Amin M, Kinsella J: Patient mobile telephone 'text'reminder: a novel way to reduce non-attendance at the ENT out-patient clinic. J Laryngol Otol 2008, 122(03):296-298.

doi:10.1186/1472-6947-13-127

Cite this article as: Huang et al.: Effects of and satisfaction with short message service reminders for patient medication adherence: a randomized controlled study. BMC Medical Informatics and Decision Making 2013 13:127.

\section{Submit your next manuscript to BioMed Central and take full advantage of:}

- Convenient online submission

- Thorough peer review

- No space constraints or color figure charges

- Immediate publication on acceptance

- Inclusion in PubMed, CAS, Scopus and Google Scholar

- Research which is freely available for redistribution

Submit your manuscript at www.biomedcentral.com/submit
C Biomed Central 